\title{
BMJ Open A protocol for engaging unlicensed private drug shops in Rural Eastern Uganda for Integrated Community Case Management (ICCM) of malaria, pneumonia and diarrhoea in children under 5 years of age
}

\author{
Denise L Buchner, ${ }^{1}$ Phyllis Awor ${ }^{2}$
}

To cite: Buchner DL, Awor P. A protocol for engaging unlicensed private drug shops in Rural Eastern Uganda for Integrated Community Case Management (ICCM) of malaria, pneumonia and diarrhoea in children under 5 years of age. BMJ Open 2015;5:e009133.

doi:10.1136/bmjopen-2015009133

\section{- Prepublication history} and additional material is available. To view please visit the journal (http://dx.doi.org/ 10.1136/bmjopen-2015009133).

Received 19 June 2015 Revised 1 September 2015 Accepted 10 September 2015

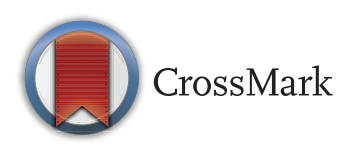

${ }^{1}$ Faculty of Medicine, University of Calgary, Calgary, Alberta, Canada ${ }^{2}$ Department of Public Health, Makerere University, Kampala, Uganda

Correspondence to Dr Denise L Buchner; dlbuchne@ucalgary.ca

\section{ABSTRACT}

Introduction: Malaria, pneumonia and diarrhoea are leading causes of death in young children in Uganda. In 2010, Integrated Community Case Management (ICCM) was adopted in Uganda for community level diagnosis and treatment of these diseases through community health workers. However, $50-60 \%$ of sick children will receive treatment from the private sector, especially drug shops. Only about half of drug shops are licensed and the quality of care is poor. There is an urgent need to improve quality of care and regulation of drug shops in Uganda.

Methods: This is a pre-post cross-sectional study with before and after measurement in an intervention area in Kamujli district. A snowball mapping exercise, exit interviews, focus group discussions and interviews will be used. 25 randomly selected drug shops will be selected for an intervention that will assist drug shops to become licensed, and provide five days of ICCM training, subsidised prepackaged medicines (artemisinin-based combination therapies for malaria, amoxicillin for pneumonia, Oral Rehydration Salts/zinc for diarrhoea) and free diagnostic tools (rapid diagnostic tests, respiratory timers, thermometers, algorithms). We anticipate a sample size of 1200 (600 at baseline and 600 at the end of the study).

Analysis: Quantitative data will be analysed using SPSS for proportions and Cls. Bivariate and multiple logistic regression analysis with adjustment for clustering of data will be performed to adjust for confounding and determine intervention effect. Qualitative data will be entered into NVivo 10 and analysed using content analysis.

Ethics and Dissemination: Research ethics approval is received from the University of Calgary (REB 14-0269), and Makerere University (IRB00011353). Findings from this study will be disseminated through journal articles and conference presentations, and will illustrate the feasibility of introducing ICCM for drug shops.

\section{Strengths and limitations of this study}

- Integrated Community Case Management (ICCM) delivered through private drug sellers might be a more sustainable model for delivery of life-saving medicines.

- No study to date has examined ICCM delivered through unlicensed drug sellers. Few studies have gathered data from unlicensed drug sellers for any purpose.

- This study will develop a model for licensing and regulating unlicensed drug shops.

- Drug shops in our intervention will become licensed before they begin drug distribution. Our agreement with the National Drug Authority does not permit us to distribute drugs to unlicensed drug shops.

- As we are only able to carry out our intervention with drug shops that become licensed, we are limited to a pre-post cross-sectional study design.

\section{BACKGROUND}

Worldwide the number of children who die has decreased, although this decrease has been slow and in some countries, particularly in sub-Saharan Africa, the decrease has stagnated or even reversed. ${ }^{1}$ In developing countries, pneumonia, malaria and diarrhoea remain the main causes of mortality for children below 5 years of age. ${ }^{2}$ Even though effective and inexpensive treatments are available, many children in Africa, and especially those who live in rural or hard-to-reach places, do not receive life-saving treatments for common and easily treatable childhood illnesses. $^{3-6}$

Early and accurate treatment of pneumonia, malaria and diarrhoea is essential to 
prevent mortality, avert complicated illnesses and reduce irrational use of drugs. ${ }^{1}{ }^{3}{ }^{4}$ Integrated community case management (ICCM) of childhood illnesses is a taskshifting strategy that extends the management of common childhood illnesses beyond health facility care so that more children have access to lifesaving medicines. In many cases, ICCM is delivered through lay community members who receive basic training.

In many developing countries, treatment levels for common illnesses are unacceptably low, especially in rural and remote communities. ${ }^{1}$ In sub-Saharan Africa, the WHO reports that only $39 \%$ of children with diarrhoea receive ORS (Oral Rehydration Salts) and 30\% of children with presumed pneumonia receive antibiotics. Moreover, WHO reports that less than $20 \%$ of sub-Saharan children with fever receive artemisininbased combination therapy (ACT). ${ }^{1}$

Nearly a decade of research on ICCM has demonstrated that ICCM projects that are supported, supervised and have a reliable supply of drugs can significantly reduce child mortality ${ }^{1} 7$ In Malawi, Theodoratou $e t a l^{8}$ estimate that ICCM for pneumonia can reduce under-five mortality by $70 \%$. In Burkina Faso, Sirima et at found that ICCM of malaria resulted in a $40 \%$ reduction in malaria-specific mortality and a $60 \%$ overall reduction in child mortality. Additionally, Munos $e t a l^{10}$ report that ORS delivered through ICCM can prevent $70-90 \%$ childhood mortality caused by acute watery diarrhoea.

Until recently, ICCM has been carried out almost exclusively through public sector models, and primarily through community health workers (CHW). Although standards for CHWs differ between countries, CHWs are often lay volunteers, sometimes with low levels of education, who receive basic training (2 weeks- 1 year) and are supplied with prepackaged medicines (and sometimes diagnostics) for common childhood illnesses. ${ }^{11}$ Despite widespread recognition of the major impact ICCM programmes have on reduced child mortality, many countries are unable to sustain or scale-up ICCM through CHW programmes once funding is withdrawn.

In many countries, private sector health providers, including informal drug shops, are routinely consulted when illness strikes. ${ }^{11} 12$ The WHO estimates that between $30 \%$ and $70 \%$ of febrile children are treated in the private sector, including drug shops. Other studies report that between $39 \%$ and $53 \%$ of people who seek treatment for an illness consult a private health provider first, including general merchandise sellers, pharmacies, for-profit providers and drug sellers. ${ }^{13-15}$ In areas where access to formal health facilities is difficult, private health providers are often the first point of entry into the healthcare system, often because the care is accessible, affordable and perceived as patient-friendly. ${ }^{16}$

Despite widespread utilisation of private sector healthcare providers, knowledge about the sector is extremely limited. What is known, however, is that many private sector providers are unlicensed and unsupervised. They often diagnose illnesses incorrectly, sell truncated doses of medicines, expired drugs and drugs that are not recommended by national guidelines. ${ }^{17}$ There is a need to improve the quality of care provided by private sector health providers and discover strategies to regulate their practice. ${ }^{13-16} 18-20$

Recently, the WHO has recognised the major role that private sector health providers play in health provision, and the possibility of tapping into this reserve of health providers as a potential source for ICCM. ${ }^{11}{ }^{12}$ To date, few studies have examined ICCM by private health providers, and none have examined ICCM by unlicensed private health providers.

\section{Ugandan context}

In Uganda, under 5 years mortality rate remains high at $90 / 1000,{ }^{21}$ while the infant mortality rate is $54 / 1000 .{ }^{21}$ The Millennium Development Goal to reduce under 5 years mortality rate to $56 / 1000$ by 2015 (MDG 4) will not be reached. ${ }^{22}$ Under 5 years mortality rate in Uganda is caused primarily by pneumonia, diarrhoea and malaria. ${ }^{23}$ In rural areas, treatment for common childhood diseases are often inaccessible due to the long distances to health facilities, and few or no trained health professionals available at the community level. These challenges are compounded by other factors including delayed healthcare seeking, poor knowledge about danger signs, irrational use of drugs, clinical overlap of other conditions, comorbid factors, low rates of immunisation, high levels of malnutrition, underdosing, low quality of drugs, and poorly trained health providers. $^{24}$

Utilisation of public health facilities in Uganda is low. Konde-Lule $e t a l^{4}$ found that $42 \%$ of persons interviewed $(\mathrm{n}=2580)$ reported being ill in the past month, but only $54.1 \%$ sought care from any type of health provider. Of these, $37 \%$ visited a public health facility and $39 \%$ sought care from a private for-profit provider, often an individually owned clinic or drug shop. Similar results were found by Awor et al, ${ }^{13}$ who report that $53 \%$ of febrile children were taken to a private for-profit health provider. The most commonly cited reasons for seeking health services from community-based providers are convenience, and perceived severity of the illness. ${ }^{17} 1925-27$

Despite high utilisation rates, health services offered by private drug shops in Uganda are entirely unsupervised; there are no minimum standards for training, no opportunities for upgrading skills and no data management requirements. ${ }^{25}$ One study conducted exit interviews at licensed private drug shops in rural Uganda and found that only $10.3 \%$ of febrile children were given appropriate antimalarial treatment while $15.6 \%$ of children with cough and fast breathing received antibiotics. Moreover, only $14.3 \%$ of children who presented with diarrhoea received ORS, and none received both ORS and zinc. ${ }^{13}$ Many studies call for interventions to regulate private pharmacies and drug shops, and discover strategies to integrate them into the health system. ${ }^{13-15} 182027$ 


\section{The intervention}

This study will use a snowball technique to map licensed and unlicensed drug shops in four subdistricts (Bolowoli, Buransi, Kitayunjwa, Namasagali) of Kamuli district in Eastern Uganda in order to determine distribution of drug sellers and distances from public health facilities. Twenty-five drug shops that are at least $2 \mathrm{~km}$ from a public health facility and which meet the criteria to become licensed will be selected for a baseline study. All drug shops that participate in the baseline study will be assisted to become licensed shops and invited to participate in the intervention. In order to assist drug sellers to license their drug shops, the study will contribute half of the licensing fee (approximately 60000 UGX) and help drug sellers to navigate the licensing process. The intervention will train up to two drug sellers per intervention drug shop in ICCM and assist drug sellers to procure subsidised prepackaged medicines for distribution to children under 5 years of age who are diagnosed with malaria, pneumonia and/or diarrhoea. Intervention drug sellers will also be provided with free diagnostic tools (thermometers, rapid diagnostic test, breathing rate monitors and diagnostic algorithms/charts). Data will be collected at baseline and at the end of the study through drug shop surveys, exit interviews, focus group discussions (FGD) and interviews.

\section{OBJECTIVES}

\section{Overarching objective}

To determine if ICCM, when introduced at intervention private drug shops, can influence appropriate treatment for children less than 5 years of age for common childhood diseases in Uganda.

\section{Specific objectives}

1. To determine the distribution of drug shops in four subdistricts of Kamuli district

2. To determine current unlicensed drug seller knowledge and treatment practices in management of fever, cough, and diarrhoea in children under 5 years of age

3. To introduce prepackaged drugs (ACTs, amoxicillin, ORS/zinc) and diagnostics (malaria rapid diagnostic tests, RDTs, respiratory timers, thermometers, treatment algorithms/charts) at intervention drug shops for management of malaria, pneumonia and diarrhoea in children less than 5 years of age

4. To evaluate the intervention for influence on drug seller knowledge and practice in the treatment of malaria, pneumonia and diarrhoea in children less than 5 years of age

5. To evaluate the intervention for influence on drug seller ability to recognise danger signs in children under 5 years of age

6. To understand the benefits and limitations of implementing ICCM in private drug shops from the perspective of key stakeholders
7. To explore possible linkages between unlicensed drug shops and the public health sector, including models for registration of drug shops.

\section{OUTCOMES}

\section{Primary outcome}

Increased accurate treatment for children under 5 years of age who are brought to an intervention drug shop for treatment of fever, cough and diarrhoea.

\section{Secondary outcomes}

1. Increase to $60 \%$ the use of RDT's to determine malaria status of children under 5 years of age who are brought to an intervention drug shop with fever

- Numerator: number of children U5 brought to an intervention drug shop with fever and who receive an RDT

- Denominator: number of children U5 brought to an intervention drug shop with fever

2. Increase to $75 \%$ accurate treatment of malaria based on RDT result

- Numerator: number of children U5 brought to an intervention drug shop with fever who have a positive RDT result and receive the correct dose of Coartem as per age of the child

- Denominator: number of children U5 brought to an intervention drug shop with fever who have a positive RDT result

3. Increase to $60 \%$ the ability of drug sellers to diagnose pneumonia using breathing rate monitors

- Numerator: number of children brought to an intervention drug shop with cough and fast breathing, and diagnosed to have pneumonia using a breathing rate monitor

- Denominator: number of children brought to an intervention drug shop with cough and fast breathing

4. Increase to $75 \%$ accurate treatment of pneumonia by drug sellers

- Numerator: number of children brought to an intervention drug shop with cough and fast breathing, and diagnosed to have pneumonia using a breathing rate monitor and who receive the correct dose of amoxicillin as per the age of the child

- Denominator: number of children brought to an intervention drug shop with cough and fast breathing, and diagnosed to have pneumonia using a breathing rate monitor

5 . Increase to $75 \%$ accurate treatment of diarrhoea by drug sellers

- Numerator: number of children brought to an intervention drug shop with diarrhoea and receive ORS and zinc

- Denominator: number of children brought to an intervention drug shop with diarrhoea 
6. Increase to $85 \%$ the ability of drug sellers to recognise danger signs in young children

- Numerator: number of intervention drug sellers participating in end of study FGD or interviews who self-report improved ability to recognise danger signs in children U5

- Denominator: number of intervention drug sellers participating in end of study FGDs or interviews

7. Improved understanding among stakeholders (health officials, drug sellers, caregivers) of the benefits and limitations of implementing ICCM in private drug shops.

\section{METHODS/DESIGN}

\section{Study design}

We will use a pre-post cross-sectional study design with before and after measurements to determine if ICCM implemented in intervention drug shops influences accuracy of treatment for children under 5 years of age with fever, cough and diarrhoea. This study design will provide insights into how our intervention influences a drug seller's practice before and after the intervention. We will collect data from drug sellers and caregivers of young children from mixed data sources (GPS data, surveys, exit interviews, FGDs and interviews) in order to illuminate current demographics and treatment practices of private drug sellers, and opportunities available for improvement. Outcomes will be measured from baseline and end of study exit interview data, FGDs and interviews. Data from this study will increase knowledge about unlicensed drug shops distribution and practices, and provide important information about how unlicensed drug shops might be engaged in the public health system.

\section{Study population}

The population of Kamuli district is approximately 5008000 . The four subdistricts selected for the study are Bolowoli (population: 54 092), Kitayunjwa (population: 55 248), Namasagali (population: 37524 ), and Butansi (population: 29 799), and these were selected due to high levels of poverty and poor access to health facilities. Most families in Kamuli district rely on subsistence farming for survival. The area is primarily rural with few roads. Most people in the area have no access to electricity, running water, or a modern sanitation system. Access to government health facilities is limited.

\section{Inclusion criteria and recruitment}

Drug sellers will be eligible to participate in the study if they own the drug shop and are the primary drug sellers in the shop. A drug shop included in the study must be at least $2 \mathrm{~km}$ from a public health facility and the shop must be free from pests, have windows or vents that open, have clean walls and floor and must have a ceiling. Drug sellers must also be willing to meet the criteria to become a licensed drug seller.
Caregivers who seek treatment from a study drug seller for a child under 5 years of age will be approached for an exit interview by a research assistant after the caregiver has received treatment for the child. Eligible caregivers must be 18 years of age or older.

\section{Consent}

Written consent will be obtained from all study participants and for all study phases. Drug shops that are identified during the mapping exercise but are closed will have their GPS information recorded and consent will not be obtained. Details in the study consent form will be explained clearly to all study participants. Study participants who are unable to write their signature will indicate consent through a thumbprint. Consent forms will be available in English and Lusoga.

\section{Sampling}

Mapping

A snowball technique will be used to map located drug shops (licensed and unlicensed) in the four selected subdistricts. Data collection will begin by obtaining village lists from local authorities. Starting at the edge of the first subdistrict, researchers will ask villagers to direct the study team to a nearby village. Once in the village, the researcher will ask for directions to the nearest drug shop. When the researcher feels that all drug shops in a village have been located, the researcher will ask for directions to the next village. GPS coordinates will also be recorded for all public health facilities located in the mapping of the subdistricts.

\section{Intervention}

Intervention drug shops will be selected from the database of all mapped drug shops in two subdistricts if inclusion criteria (ie, clean floor, walls, ceiling, windows that open, willingness to be licensed and willingness to become licensed) are met. A maximum of 25 drug shops will be selected.

Exit interviews will be conducted outside the study drug shop by trained research assistants and will continue until 25 exit interviews are collected from each drug shop. All caregivers who seek care from a drug seller for a child under 5 years of age during baseline and end of study data collection will be invited to participate in an exit interview. We anticipate our sample size for exit interviews to be 600 exit interviews at baseline and $600(\mathrm{n}=1200)$ at the end of the study based on a 95\% level of significance, $5 \%$ confidence limits and a design effect of 1.5. Final end of the study sample size will be based on proportions of correct treatment at baseline and $85 \%$ power to detect differences.

At the end of the study, FGDs will be conducted with study drug sellers and individual interviews will be conducted with key stakeholders. FGDs and individual interviews will continue until saturation of study themes is reached. 


\section{DATA COLLECTION}

\section{GPS data}

GPS coordinates will be recorded for all drug shops and health facilities located during the mapping exercise. GPS data will illustrate the distribution of drug shops (licensed and unlicensed) in relation to each other and public health facilities.

\section{Surveys}

Surveys developed for this study were adapted from the Demographic and Health Survey Questionnaires, ${ }^{28}$ and other validated tools used to collect similar data from licensed drug shops in Uganda. ${ }^{13} 29$

\section{Mapping surveys}

The mapping survey (see online supplementary appendix A) will ask about demographic information, years of operation, licensing status, drug seller ICCM knowledge, physical condition of the shop and drugs for sale. It will also include qualitative questions about barriers to licensing, support required to facilitate licensing and other challenges associated with operating a drug shop.

\section{Drug shop surveys}

Comprehensive drug shop survey (see online supplementary appendix B) data will be collected from intervention drug shops selected to participate in the intervention and each drug seller will be asked about demographic information, ownership, hours of operation, distance to nearest public health facility, record keeping, procurement, decision-making, referral practices, ICCM knowledge, disposal of expired drugs, other items for sale and physical characteristics of the drug shop. A comprehensive list of all ICCM medicines available for sale in the drug shop on the day that the survey is administered will be recorded.

\section{Exit interview surveys}

Exit interview surveys (see online supplementary appendix C) will collect data from caregivers who are 18 years of age or older, and have consulted with an intervention drug seller for treatment of a sick child who is less than 5 years of age. Exit interview surveys will ask respondents about demographic information, if the child was present when treatment is sought, symptoms present at the time of consultation, time since the onset of illness, other treatments sought, treatments prescribed including brand name, dosage received and formulation of medicine given (tablets, syrup, suspension), amount paid, instructions provided by the drug seller, method of transportation, motivation, perceived quality of drugs sold and distance to the nearest health facility. The exit interview survey will also ask caregivers if the drug seller assessed the child using any medical investigations (ie, RDT, thermometer, scale, breathing rate monitor). If the child is present during an exit interview and is reported to have fever, the research assistant will obtain the child's temperature, conduct an RDT for malaria and refer children with a positive test result to a public health facility. Trained research assistants will wait outside the intervention drug shops from 8:30 to 17:00 daily to conduct exit interviews, until at least 25 surveys are collected from each drug shop.

\section{FGD and interviews}

FGD with drug sellers

FGDs will be conducted with intervention drug sellers at the end of the study. Each FGD will consist of 4-6 participants and will ask drug sellers about their involvement with the study, the impact the study had on their business, what could be improved in future interventions and the likelihood that the drug seller will continue to provide interventions introduced during the study.

\section{Interviews with key informants}

At the end of the study, individual interviews will be conducted with key informants. Key informants will include individuals who were involved with the study, including representatives from the National Drug Authority and the Ugandan Ministry of Health. Key informant interviews will ask informants about their involvement with the study, opinions regarding implementing ICCM in private drug shops and opportunities for scale-up of the project.

\section{DATA ANALYSIS PLAN}

Data analysis for this study will be descriptive using a combination of qualitative and quantitative methods.

\section{Surveys}

Mapping surveys

GPS mapping data collected during the mapping surveys will be plotted on Google Maps in order to visualise distribution of drug shops, and the distances to public health facilities.

Other mapping survey data will be analysed using both quantitative and qualitative analysis strategies.

\section{Drug shop surveys}

Quantitative analysis of drug shop surveys will be performed to identify frequencies, cross tabulations and ratios of drug seller characteristics and practices.

\section{Exit interview surveys}

Quantitative analysis of exit interview surveys will be performed to describe use of diagnostic tools and treatment practices by drug sellers. We will provide detailed tabulations of responses by child characteristics (age, gender), symptoms for which treatment was sought (fever, cough, diarrhoea) and treatments received. The significance of observed differences will be evaluated using $\chi^{2}$ tests.

\section{FGDs and key informant interviews}

FGDs and key informant interviews will be audio recorded, transcribed verbatim, translated to English, 
assigned a unique identifier and imported into NVivo 10 , and cross-checked by a second researcher. Analysis will employ a descriptive phenomenology approach as described by Kleiman ${ }^{30}$ to discover meaning from lived experience. Data analysis strategies will include becoming familiar with data, bracketing, identification of an emerging thematic framework, discovering the essential meaning of the outcome, and description of discovered phenomenon. Memos, word clouds and models will be used to describe and illustrate meaning garnered from the data. Results will be cross-checked by a second qualitative investigator.

\section{Relevance of findings}

Worldwide child mortality from malaria, pneumonia and diarrhoea remains high. Inexpensive and effective treatments are available, but distributing these treatments to children in rural areas is challenging. Engaging private drug sellers to diagnose and treat common childhood diseases might be a sustainable method of ICCM delivery to reduce child mortality. The strategy might also encourage regulation and registration of drug shops.

Contributors DLB is the principle investigator on the Grand Challenges Grant. DLB and PA conceptualised the project and developed the study proposal design. DLB and PA supervised the study. DLB and PA drafted the manuscript.

Funding This work is supported by Grand Challenges Canada Stars in Global Health Round Six (\#0492-01-10).

Competing interests None declared.

Patient consent Obtained.

Ethics approval University of Calgary; Makerere University.

Provenance and peer review Not commissioned; externally peer reviewed.

Open Access This is an Open Access article distributed in accordance with the Creative Commons Attribution Non Commercial (CC BY-NC 4.0) license, which permits others to distribute, remix, adapt, build upon this work noncommercially, and license their derivative works on different terms, provided the original work is properly cited and the use is non-commercial. See: http:// creativecommons.org/licenses/by-nc/4.0/

\section{REFERENCES}

1. Young M, Wolfheim C, Marsh D, et al. World Health Organization/ United Nations Children's Fund joint statement on integrated community case management: an equilty-focus strategy to improve access to essential treatment services for children. Am J Trop Med Hyg 2012;87:6-10.

2. WHO. Integrated management of childood illness-handbook. Geneva: World Health Organization, 2005.

3. Schellenberg JA, Victora CG, Mushi A, et al. Inequalities among the very poor: health care for children in rural southern Tanzania. Lancet 2003;361:561-6.

4. Victoria CG, Wagstaff A, Schellenberg J, et al. Applying an equality lens to child health and mortality: more of the same is not enough. Lancet 2003;362:233-41.

5. Gyapong M, Garshong B. Lessons learned in home management of malaria: implementation research in four African countries. Geneva: World Health Organization, 2007.

6. Yeboah-Antwi K, Pilingana P, Macleod W, et al. Community case management of fever due to malaria and pneumonia in children under five in Zambia: a cluster randomized controlled trial. PLoS Med 2010;7:e1000340.

7. Lainex $Y B$, Wittcoff $A$, Mohamud $A I$, et al. Insights from community case management data in six sub-Sarahan African countries. Am Trop Med Hyg 2012;87(5 Suppl):144-50.

8. Theodoratou E, Al-Jilaihawi S, Woodward F, et al. The effect of case management on childhood pneumonia mortality in developing countries. Int J Epidemol 2010;39(Suppl 1):i155-71.

9. Sirima SB, Konaté A, Tiono A, et al. Early treatment of childhood fevers with pre-packaged antimalarial drugs in the home reduces sever malaria morbidity in Burkina Faso. Trop Med Int Health 2003;8:133-9.

10. Munos MK, Walker CL, Black RE. The effect of oral rehydration solution and recommended home fluids on diarrhea mortality. Int $J$ Epidemol 2010;39(Suppl 1):i75-87.

11. D'Acremond V, Bosman A. WHO informal consultation on fever management in peripheral health care settings: a global review of evidence and practice. Global Malaria Programme, WHO, Geneva 2013.

12. Rutebemberwa E, Kadobera D, Katureeb S, et al. Use of community health workers for management of malaria and pneumonia in urban and rural areas in eastern Uganda. Am J Trop Med Hyg 2012;87(5 Suppl):30-5.

13. Awor $\mathrm{P}$, Waman $\mathrm{H}$, Bwire $\mathrm{B}$, et al. Private sector drug shops in integrated community case management of malaria, pneumonia, and diarrhoea in children in Uganda. Trop Med Hyg 2012;86:92-6.

14. Konde-Lule J, Gitta SN, Lindfors A, et al. Private and public health care in rural areas of Uganda. BMC Int Health and Human Rights 2010;10:29.

15. Konde-Lule J, Okuonzi S, Matsiko C, et al. The potential of the private sector to improve health outcomes in Uganda. Makere University, 2006.

16. Buchner D. Stories without endings: illness and disability narratives from Lao PDR. Calgary: University of Calgary, 2011.

17. Bryce J, Victora C. Ten methodological lessons from the multi-country evaluation of integrated management of childhood illness. Oxford University Press, 2005.

18. Nabudere $\mathrm{H}$, Asiimwe D, Mijumbi R. Task shifting in maternal and child health care: an evidence brief for Uganda. Int $J$ Technol Assess Health Care 2011;27:173-9.

19. Rutebemberwa E, Pariyo G, Peterson S, et al. Utilization of public or private health care providers by febrile children after user fee removal in Uganda. Malar J 2009;8:45.

20. Wiysonge C. Can working with private for-profit providers improve utilization and quality of health services for the poor? A SUPPORT summary of a systematic review. 2008. http://wwwsupportcollaborationorg/summarieshtm

21. Ugandan Bureau of Stastics (UBOS) and ICU International INC. Uganda demographic and health survey 2011. Kampala, Uganda: UBOS and Calverton, Maryland: ICF International Inc., 2012.

22. MofH. Integrated community case management of childhood malaria, pneumonia and diarrhea implementation guidelines. Ministry of Health, Kampala 2010.

23. Ministry of Health. Uganda health sector strategic \& investment plan 2010/11-2014/15. Ministry of Health, 2010.

24. Graham S, English M, Hazir T, et al. Challenges to improving case management of childhood pneumonia at health facilities in resource-limited settings. Bull World Health Organ 2008;86:349-55.

25. Bryce J, Victora C, Habicht J, et al. Programmatic pathways to child survival: results of a multi-country evaluation of integrated management of childhood illness. Oxford University Press, 2005.

26. Victora C, Schellenberg J, Huicho L, et al. Context matters; interpreting impact findings in survival evaluations. London: Oxford University Press, 2005.

27. Rutebemberwa E, Kallander K, Tomson G, et al. Determinants of delay in care-seeking for febrile children in eastern Uganda. Trop Med Int Health 2009;14:472-9.

28. ICF International. Demographic and Health Survey Methodology Questionnaires: Household, woman's, and man's. Measure DHS Phase III: Calverton, Maryland, USA. http://www.measuredhs.com/ publications/publication-DHSQ6-DHS-Questionnaires-and-Manuals $\mathrm{cfm}$

29. Awor $\mathrm{P}$, Wamani $\mathrm{H}$, Tylleskar $\mathrm{T}$, et al. Drug seller adherence to clinical protocols with integrated management of malaria, pneumonia and diarrhoea at drug shops in Uganda. Malar $J$ 2015;14:277.

30. Kleiman S. Phenomenology: to wonder and search for meaning. Nurse Res 2004;11:7-19. 